\title{
«NUEVOS» $\mathrm{Y}$ «VIEJOS» PARADIGMAS EN LA SOCIOLOGAA DE LA EDUCACIÓN. ANÁLISIS DEL PROCESO BRITÁNICO
}

\author{
Lluís Samper \\ (Universitat Autonoma de Barcelona)
}

\section{INTRODUCCION}

Es sabido que el desarrollo de una disciplina científica lejos de presen. tar una evolución lineal y acumulativa, como pudiera sugerit la lectura de manuales y libros de texto, presenta tanto estadios de crecimiento y consolidación como fases de crisis, de estancamiento y confusión. Tal vez sea éste el caso de la sociología de la educación, que después de dos o más décadas de expansión cuantitativa y fecundidad de perspectivas teóricas parece ha. ber llegado a cierta situación de saturación y empantanamiento. Para Alonso (1983, p. 166) el fenómeno es prácticamente universal y obedecería, entre otras razones, a una serie de rupturas en el seno de la propia área de conocimiento: entre generaciones, entre posiciones ideológicas, entre países y entre los especialistas ubicados en los departamentos universitarios y en centros de formación de profesores.

En el caso concreto de España, es obvio que la corriente de expectativas positivas generada en los setenta no se ha visto en absoluto confirmada. No sólo la sociología de la educación española está aún lejos de su instituciona. lización sino que, paradójicamente, da muestras de cierto cansancio y decepción (Alonso, 1983, p. 175). Existe entre los especialistas un consenso generalizado sobre las miserias de la situación presente, definida como situación de impasse (Subirats, 1983), decepción (Alonso, 1983), fatalismo y desencanto (March, 1984)... ya anticipada por el falso esplendor de los setenta (Ortega, 1983). En cuanto a las tazones explicativas de tan confusa 
e incierta situación, los diagnósticos, pese a su diversidad, pueden ser resumidos, en mi opinión, en los siguientes:

a) Problemas teóricos: agotamiento de los paradigmas clásicos y, más concretamente, excesivo énfasis en las teorías mactosociológicas sobre la teproducción social y, casi exclusivamente, en lengua francesa (Ortega, 1983, p. 367; March, 1984); circularidad de los tazonamientos y teiteración de las argumentaciones (Subirats, 1983, p. 81).

b) Problemas institucionales: problemas de definición epistemológica (Quintana, 1980; Lerena, 1983), raquitismo del marco académico unido a una absurda proliferación de congresos, reuniones, jornadas, etc. (Subirats, 1983; Alonso, 1983; Quintana, 1983...), gremialismos y corporativismo (Ortega, 1983), agtavados por tendencias de «capillismo» ideológico, etc.

c) Problemas estructurales: atonía de la educación y desmovilización de las ideologías educativas (Subirats, 1983), repercusiones de la crisis económica (reducción de los presupuestos destinados a investigación educativa, congelación de plantillas de profesores...), bloqueo de los proyectos destinados a actualizar la formación de docentes, arcaísmo y resistencia al cambio en la Universidad.

Con respecto al tema de los problemas de definición epistemológica conviene precisar que la controversia no es meramente teórica sino que, como suele suceder en el seno de las comunidades científicas, aparece envenenada por intereses gremialistas y antagonismos ideológicos. Como es sabido, la polémica sobre la delimitación del área de conocimiento correspondiente a sociología de la educación se traduce, de hecho, en una guerra entre sociólogos y pedagogos sobre sus tespectivas competencias profesionales. Las coincidencias histórico-estructurales del naciniento de la pedagogía social (Paul Natorp) y de la sociología de la educación (Émile Durkheim), unidas a sus diferencias epistemológicas (enfoque flosófico-normativo versus perspectiva empírica-positiva) (Sanvicens, 1969, p. 481) añaden, por así decirlo, leña al fuego.

La distinción entre ambas disciplinas patece telativamente sencilla a partir de la diferenciación entre objeto material (o real) y objeto formal (o de conocimiento). En este sentido, pedagogía y sociología de la educación tienen en común su objeto material, que es la educación, pero differen en cuanto a su objeto formal $o$, dicho de otro modo, en la perspectiva desde la que lo estudian (Quintana, 1980, p. 36; Lerena, 1983, p. 13). Pero las cosas se complican cuando de las ciencias "puras» pasamos a las ciencias "aplicadas»; cómo, por ejemplo, se discute a nivel de la formación de maestros. Surgen entonces las agrias y mutuas acusaciones de «gremialismo» (Quinta+ 
na, 1983; Ortega, 1983), afloran las distintas concepciones ideológicas y los miembros de las variopintas tribus en que aparece dividida nuestra enseñanza superior se aprestan para el combate. Atrás quedaron los buenos propósitos de interdisciplinariedad.

Frente a quienes alegan como causa del atraso de nuestras disciplinas científicas, especialmente en los campos de las ciencias sociales y de las ciencias de la educación, una excesiva dependencia de autores y teorías foráneos, mi posición personal es que tal colonialisno cultural es más aparente que real. En efecto, como ya he comentado anteriormente, la sociología de la educación española se caracteriza por una aburrida repetición de teorías macrosociológicas centradas, obsesivamente, en temas de reproducción cultural y control ideológico. Junto a esta influencia excesiva, en mi opinión, de la sociología francesa (Bourdieu, Althusser, Baudelot...), la sociología en lengua inglesa, especialmente la "New Sociology of Education", ha recibido proporcionalmente mucha menot atención (con excepción del caso aislado, usualmente caricaturizado, de Basil Bernstein).

En el presente estudio ${ }^{1}$ pretendo presentar una rápida panotámica de la sociología de la educación británica con un doble objetivo. En ptimer lugar, con el propósito de difundir nuevos paradigmas alternativos que ayuden a revitalizar nuestra narcisista y reiterativa situación actual. Como segunda intención, mi exposición pretende, a través del análisis del proceso en Gran Bretaña, detectar las claves que conduzcan a una institucionalización de la sociología de la educación en nuestro propio país.

Obviamente, una presentación mínimamente rigurosa de la situación actual de la sociología de la educación británica, así como de las etapas del proceso conducente a dicha situación, tebasa notablemente la extensión del presente ensayo y, seguramente, las posibilidades de su autor. Mis pretensiones son mucho más modestas y se circunscriben a ofrecer una visión panorámica, espero que no sesgada por prejuicios ideológicos o de otro tipo, de este tema.

1. El presente trabajo ha sido posible merced a una beca de ampliación de estudios en el extranjero concedida por la CIRIT de la Generalitat de Catalunya (CIRIT. EE8312-91). También deseo expresar mi agradecimiento al doctor Bill Williamson, de la Universidad de Dutham, por sus valiosos consejos para ta redacción del mismo si bien, evidentemente, me considero el único responsable de sus limitaciones y defectos. 


\section{LA SOCIOLOGIA DE LA EDUCACION EN LA GRAN BRETAÑA}

\subsection{Procesos de institucionalización: funcionalismo $y$ polítical atithmetic}

A diferencia de los EE.UU., cuyas primeras producciones en este campo datan de principios de siglo, la sociología de la educación británica inicia sus procesos de institucionalización a partir de la Segunda Guerra Mundial. Para Szreter $(1960$, p. 175$)$, en dichos procesos es fundamental la existencia de una revista realmente especializada que, aprobando o denegando el acceso a la misma a los miembros de la comunidad científica, contribuya a la lucha en pro de la legitimación de una nueva área de conocimiento. En este sentido, mientras el americano "Journal of Educational Sociology" data de septiembre de 1927 (asumiendo en 1963 el significativo cambio de título "Sociology of Education»), hasta mazzo de 1980 no aparecerá el «British Journal of Sociology of Education».

No obstante existen, según este mismo autor, otros parámetros del proceso de institucionalización, como son: 1) la aceptación de los nuevos contenidos por parte de la educación superior, especialmente, por parte de las universidades; 2) la aparición de un grupo de profesionales especializados, aceptados como tales y con goce de la autonomía suficiente, y 3) el reconocimiento de la nueva átea por parte de los organismos públicos; más específicamente, de los organismos gubernamentales. Obviamente, no hace falta insistir en la importancia crucial de que las universidades legitimen mediante sus curricula, titulaciones, programas de investigación, etc., la nueva área de conocimiento.

En el caso británico, el relativo retraso de la institucionalización de la sociología de la educación es explicable en términos del bloqueo paralelo del desarrollo de la «ciencia-madre», vale decir, la sociología. A su vez dicho bloqueo puede ser analizado a partit de la oposición de otras ciencias sociales (especialmente la antropología social), del arcaísmo de las instituciones universitarias, etc. Sea como fuere, la mayoria de los autores (Banks, 1982; Bernstein, 1977; Halsey, 1965; Karabel y Halsey, 1979; Shipman, 1973; Szereter, 1983; Williamson, 1974...) sitúan los inicios de la nueva disciplina en los estudios sobre movilidad social e igualdad de oportunida. des realizados en el seno de la London School of Economics por investigadores como D. Glass, J. Floud, A. H. Halsey, J. W. B. Douglas...2 Podemos

2. Las dos obras más representativas de este período son Floud, J., A. H. Halsey y F. M. Martin, Social class and Educational Opportunity, Heineman, Londres, 1956; Douglas, J. W. B., The Home and the Scbool, Mc Giblon and Kee, Londres, 1964. 
situar cronológicamente este período entre finales de los cincuenta y primeros años sesenta, si bien el despegue de la sociología, aún no subdividida en sociologías especiales, data de los años cuarenta. Peto... cy las aportaciones de K. Mannheim? Respecto a este pensador germano-británico, cuya valiosa aportación a la sociología nadie puede negar (Mannheim, 1936, 1943...), s13 influencia respecto de la sociología de la educación es asunto controvertido. Para Halsey (1965), por ejemplo, las teorías de Karl Mannhein sobre educación y sociedad tuvieron un eco muy reducido, apenas si transpasaron el reducido núcleo inicial del London Institute of Education. A diferencia de su obra capital, Ideología y utopía, donde establece las bases de la sociología del conocimiento, los escritos de Mannheim sobre educación presentan un tono normativista e idealista que explican, al menos en parte, su situación de «padre negado» de los sociólogos de la educación británicos. ${ }^{3}$

Análogamente, las aportaciones de la denominada «Educational Sociology» son mayoritariamente consideradas como irrelevantes. De hecho, incluso se discute hoy que tal disciplina, que en sus momentos de máxima vigencia era considerada como asignatura troncal en la mitad de las escuelas normales norteamericanas, corresponda propiamente al área de conocimiento de la sociología. Sus críticos (Karabel y Halsey, 1979; Szereter, 1980) le reprochan, entre otras limitaciones, su endeblez teórica, su excesivo pragmatismo y su orientación psicologista. Sea como fuere, su influjo real no llegó a rebasar el ámbito académico de los EE.UU.

Como ha indicado el propio Halsey (Halsey, 1965; Karabel y Halsey, 1979), el enfoque predominante de esta inicial sociología de la educación británica pudiera ser denominado «funcionalismo reformista». Su aparato conceptual procede de los estudios de sociología general, más concretamente de los estudios sobre estratificación social, y su objetivo prioritatio es el estudio de temas tales como el «desperdicio» de talentos, las «disfunciones» del sisțema educativo, las injusticias e ineficiencias derivadas de las desigualdades educativas... Metodológicamente su nota más característica es el uso de un gran aparato estadístico. Se trata de una perspectiva que si bien en sus fundamentos teóricos es funcionalista, posee una indudable vocación crítica y aplicada, de modo que ideológicamente está muy próxima al partido laborista (Banks, 1982; Bernstein, 1977). De ahí el énfasis en el concepto de «investigación-acción» y el compromiso personal de algunos de tales sociólogos en proyectos de reforma educativa: escuelas integradas (compre.

3. Por otra parte, no se olvide que la cátedra concedida en 1946 a K. Mannheim en el London Institute of Education tenía corno denominación «Teoría de la Educación». 
bensive schools), educación compensatoria, áreas de prioridad educativa, etc. ${ }^{4}$ Tampoco podemos ignorar, como factor relevante de esta intertelación entre la comunidad científica y la administración educativa estatal, la publicación de los informes oficiales sobre la situación educativa conocidos como «Newsom Report» (1963 y 1968) y «Plowden Report» (1967).

Para Robinson (1983), los antecedentes de esta "aritmética politica» se remontan al siglo xvir con la obra de Sir William Petty, un coetáneo y amigo de Hobbes, matemático, médico, economista y literato para quien las políticas administrativas debían estar basadas en la cuidadosa recolección de estadísticas. No obstante, no alcanzará su pleno desarrollo hasta el fabianismo decimonónico y su pretensión de que la ciencia social debe ponerse al servicio de la mejora de las condiciones de vida de la humanidad. Surge entonces el famoso concepto de la igualdad de oportunidades. Segín este mismo autor (Robinson, 1983) las dos notas características de tal political aritbmetic son:

a) Empirismo, de modo que se fundamenta en una representación estadística de la realidad social.

b) Reformismo, derivado de su compromiso moral de mejora de la sociedad.

Al constatar la ineficacia de la selección escolar («desperdicio». del talento, inexistencia de la igualdad de oportunidades, etc.) estos sociólogos propugnan una serie de medidas (desarrollo de una educación integrada, expansión de la enseñanza superior, oferta de programas de educación compensatoria, revisión de la formación de los docentes...) que, con el tiempo, irán siendo asumidas por la política gubernamental.

Bernstein (1977) nos recuerda que en los debates de esta época la idea básica era la demostración, no la explicación, de las fuentes institucionales de las desigualdades educativas. Por otra parte, la sociología de la educación aún no está institucionalizada como disciplina académica en los departamentos de educación ni, aún menos, en los de sociología. Su transformación de un campo especializado de investigación en un área de conocimiento científico fue especialmente difícil dado el bajo estatus académico del que gozaba el tema de la educación. Su irstitucionalización, a mediados de los sesenta, fue sorprendentemente exitosa tanto por las razones de coyuntura política

4. Como botón de muestra del compromiso de los sociólogos británicos con el reformismo educativo del gobierno laborista puede consuitarse: Halsey, A. H., Educational Priority: EPA Problems and Policies, Her Majesty's Stationery Office, Londres, 1972. 
anteriormente expuestas como debido a que coincidió con un momento de expansión del sistema educativo, especialmente a nivel de escuelas universitarias y facultades de educación (Bernstein, 1977, pp. 161.162; Karabel y Halseyl, 1979, p. 5; Banks, 1982 p. 20).

En efecto, en este proceso de desarrollo y consolidación de la sociología de la educación no podemos ignorar su relevancia a nivel de la formación de docentes. Ya en 1963 el «Newsom Report» tecomendaba explícitamente que la formación de maestros debetía incluir necesatiamente cursos de sociología. Por otra parte, especialistas tan acreditados como Halsey (1965) y Musgrave (1965) insistieron en la conveniencia de que los profesores desarrollaran una imaginación sociológica que les ayudara a comprender mejor su propia tarea profesional y a sus alumnos. De hecho, lo que se estaba debatiendo era una completa tedefinición del tol de maestro concebido más como un trabajador social que como un transmisor de conocimiento. Para el «James Report» (1972), específicamente destinado a actualizar la formación de los docentes, los dos objetivos priotitarios de la inclusión de la sociología de la educación en los curricula de los maestros eran: 1) capacitarios para comprender mejor los problemas educativos de las sociedades contemporáneas, y 2) que les capacitara a ellos mismos para enseñar ciencias sociales (Reid y Wormald, 1974, p. vi). La evolución del número de colleges of education que ofrecian al menos un curso de sociología es suficientemente indicativa:

\section{0: 6 colleges \\ 1964: 12 colleges \\ 1966: 28 colleges \\ 1968: 46 colleges \\ 1972: 51 colleges (aproximadamente $1 / 3$ de los colleges of education existentes).}

Esta rápida expansión aún setía incrementada durante la década de los setenta merced a la difusión de la «New Sociology of Education». Por otra parte, existe un paralelismo entre el desarrollo de la sociología de la educación en las factiltades y escuelas universitarias de educación y la expansión de la investigación y la enseñanza de la sociología dentro de las restantes facultades universitarias (Shaw, 1981, p. 19). Un análisis de contenido de los programas de los cursos de sociología impartidos en los centros destinados a la -formación de maestros (Pegram y Abell, 1974) sugiere, no obstante, que la sociología de la educación propiamente dicha ocupaba un lugar poco importante en dichos curricula. En otras palabras, se trataba más 
de una sociología para educadores que de una especialización de la sociología de la educación. ${ }^{5}$

La hiporrepresentación de la sociología de la educación pudiera imputarse, según Szereter (1983, pp. 157.158) a las diferencias de prestigio entre «sociólogos» propiamente dichos, procedentes de los departamentos de sociología, y sociólogos, en ocasiones «educadotes», que trabajan en los teachers colleges. Análogamente, un análisis de contenido de los artículos publicados entre 1953 y 1979 en las tres principales revistas británicas de sociología indica el absoluto predominio, más del doble, de las publicaciones procedentes de profesores de los departamentos de sociología frente a los procedentes de los departamentos de educación. El hecho de que fueran rechazados el $82,2 \%$ de los originales de los profesores de los centros destinados a la formación de maestros es también sumamente indicativo de hasta qué punto la posición de un científico en la estructura social de la ciencia puede afectar a la evaluación de su producción. Sobre estas diferencias de prestigio entre las distintas instituciones, y más concretamente sobre el descrédito académico del campo semántico «educación», Davies (1983, pp. 91-92) ha subrayado la paradoja de la importancia de la enseñanza/ formación de profesores como mercado de la sociología de la educación, siendo al mismo tiempo una de las fuentes permanentes de sus conflictos institucionales. Este mismo autor destaca el hecho de que en el estudio del desarrollo de la sociologia de la educación ha primado la tendencia a realizar análisis teóricos en detrimento de la investigación sobre las bases institucionales (otganizaciones en departamentos, clanes académicos...) de la disciplina.

La interdependencia entre el desarrollo de la sociología de la educación, su lucha en pos de la legitimidad académica y su utilización en la formación de profesores alcanzará su plenitud durante el período de hegemonía de la denominada «New Sociology of Education».

5. Los datos son los siguientes:

I. Áreas de conocimiento mencionadas en el $25 / 50 \%$ de los programas: Economía. Historia social. Filosofía social. Antropología social.

II. Areas de conocimiento mencionadas en el $50 / 75 \%$ de los programas: Sociología de la educación. Criminología. Sociología política.

III. Areas de conocimiento mencionadas en más del $75 \%$ de los programas: Teoría sociológica. Sociología del trabajo. Metodología. Estratificación social. Sociología rural/urbana. Estructura social y población. Psicología social. Administración educativa. 


\subsection{Orto y acaso de la «New Saciology of Education»}

La publicación en 1971 del libro editado por M. F. D. Young, Knowledge and Control, que incluye, entre otros, textos de B. Bernstein, P. Bourdieu, del propio M. F. D. Young y de sus discípulos G. M. Esland y N. Keddie significará, como es sabido, la aparición de una decisiva línea de ruptura en la evolución de la sociología de la educación británica y, al decir de algunos autores (Gorbutt, 1974; Hurn, 1976; Demaine, 1982; Forquin, $1983 \ldots$ ), el surgimiento de un nuevo paradigma teótico. ${ }^{6}$ Efectivamente, pues las diferencias entre la «nueva» y la «vieja» sociología de la educación comprenden una redefinición de los enfoques teóticos, de los temas centrales, de las metodologías y técnicas de investigación e incluso de los presupuestos antropológicos (Mascaró, 1983).

Como hemos visto anteriomente, la sociología de la educación británica de los años sesenta, si bien era de orientación socialdemócrata, reformista, a nivel teórico estaba dominada por el enfoque funcionalista. En palabras de Halsey y Karabel $(1979$, p. 11), su aparato conceptual to habían tomado prestado de las teorías funcionalistas de estratificación social... exagerando el papel de la tecnología y subestimando la importancia del conflicto y de la ideología. Por otra parte, ya hemos indicado que aunque el alcance de las desigualdades educativas estaba bien documentado, las causas de tales desigualdades petmanecían sin explicar (Bernstein, 1977; Banks, 1982).

El enfoque de la «New Sociology of Education», fenomenológico e interaccionista, busca más ofrecer explicaciones que cuantificar meramente la incidencia de las desigualdades educativas. Dicho de otro modo, el nuevo paradigma es fundamentalmente un paradigma interpretativo. Gorbutt, Young y Bernstein coinciden en denunciar el hecho de que durante demasiado tiempo los sociólogos dieran por buenas las definiciones de la problemática educativa desde el punto de vista de los administradores. Conceptos como «fracaso escolar», «buen alumno» o «educabilidad» no son categorías a priori que no puedan ser, ellas mismas, cuestionadas (Keddie,

6. En realidad, el inicio de la «New Sociology of Education» cabe situarlo en la conferencia anual de la British Sociological Association celebrada en Durham en 1970. Fue en dicha reunión profesional donde públicamente se manifestaron las diferencias entre la «nueva» y la «vieja» sociología y donde surgí́ el proyecto de editar «Knowledge and Control». Algunas de las características del nuevo paradigma (enfoques fenomenológico e interaccionista, análisis del curriculum...) ya habian sido esbozadas en las respectivas tesis doctorales de Esland y Keddie, ambas dirigidas por M. F. D. Young en la Universidad de Londres. Los títulos de ambas son suficientemente explícitos: Estand, G., «Subject and Pedagogical Perspectives in Teaching», M. A. Thesis, Univ, of London (1970); Keddie, N. «The Social Basis of Classtoom Knowledge. A case Study», M. A. Thesis, Univ. of London (1970). 
1971). Los «nuevos» sociólogos se replantean estas definiciones supuestamente basadas en el sentido común y demuestran que, de hecho, son legitimaciones producidas por el propio sistema educativo (Williamson, 1974; Hurn, 1976; Apple, 1978).

Junto a este énfasis en cuestionar las categorías pedagógicas «dadas por sentado», la «New Sociology of Education» destaca la íntima relación existente entre la estructura de conocimiento y de símbolos en las instituciones educativas y los principios y las prácticas del control social y cultural en nuestra sociedad (Davies, 1976). Coincidiendo con los pedagogos radicales americanos (Jackson, Holt) y los sociólogos neodurkheimianos franceses (Bourdieu), los «nuevos» sociólogos de la educación británicos descubren que las escuelas no sólo transmiten contenidos sino también creencias, actitudes, valores y evaluaciones. Pata decirlo en palabras de uno de sus más conspicuos representantes, empiezan a ser conscientes de que los alumnos «aprenden» en la escuela una parte significante de lo que ellos conocen como su propia "valia», su relación con los otros y con los sistemas políticos, económicos y de estratificación (Davies, 1976, p. 50). Del mismo modo que Halsey y los restantes sociólogos reformistas criticaron las insuficiencias de una política educativa liberal señalando que la mera expansión del sistema educativo no hace desaparecer las desigualdades educativas, la «New Sociology of Education» cuestiona las estrategias socialdemócratas de los años sesenta. Critican al funcionalismo reformista no sólo su fracaso relativo sino, especialmente, su endeblez teórica. Lo que les interesa a los «nuevos» sociólogọs no es tanto los determinantes sociológicos de la educación como la desmitificación de las categorías pedagógicas usuales. En este sen tido, se muestran escépticos respecto a las tenovaciones pedagógicas considerando que las desigualdades educativas sólo desaparecerán si cambia en profundidad la estructura social (Byrne y Williamson, 1975). Políticamente se sitúan en un radicalismo antiideológico y de ahí sus simpatías hacia los desescolarizadores como Illich, Reiner o Goodman.

Con respecto a las fuentes intelectuales de la «New Sociology of Education» cabe señalar dos grandes influencias (Fourquin, 1983):

a) El interaccionismo simbólico. Frente a la concepción funcionalista según la cual la educación consiste en la interiotización de normas; modelos y valores que aseguren la integración, la coherencia y la perpetuación de la sociedad, la «New Sociology of Education» se decanta decididamente hacia la sociología interaccionista de G. H. Mead. El individuo es así concebido como un actor social inmerso en un proceso continuado de comunicación. El énfasis se traslada a los conceptos de «negociación» de interpretaciones y 
«definición» de situaciones. Análogamente cambia el concepto de educación, que ahora es definida como un juego de toles abierto e improvisado.

También cabe señalar la destacada influencia de Howard Becker (1952) y sus conceptos de etiquetado, subcultura, etc.

b) La fenomenología social. Alfred Schutz había destacado el carácter subjetivamente construido de la realidad social. Esta sociología fenomenologica que considera al hombre como actor social creador de significados y no como mero producto de la sociedad tiene, a su vez, una larga tradición que nos remontaría a Max Weber, Dilthey, Simmel...

Por otra parte no podemos ignotar la decisiva importancia de la sociología del conocimiento y, muy especialmente, del conocido libro de Berger y Luckmann (1966) sobre la construcción social de la realidad.

Además de estas dos grandes corrientes teóricas, la «New Sociology of Education» se declara deudora de autores tan dispares como Marx, Mills... o, en el caso de B. Bernstein, de E. Durkheim. Esta sospechosa pluralidad de influencias condicionará, como veremos más adelante, la evolución del «nuevo' enfoque y nos permite abrigar dudas razonables sobre su carácter de paradigma altetnativo.

Además de estas importantes modificaciones teóticas los nuevos enfoques difieren de los anteriores en cuanto a los temas centrales de investigación y, muy principalmente, respecto a las metodologías y técnicas de investigación. Con respecto a los primeros cabe señalar tanto la redefinición de los temas tradicionales como la intraducción de nuevos contenidos.

Redefinición de los temas tradicionales de investigación. Según Williamson $(1974$, p. 5), aunque una primera ojeada pudiera sugerir que la "New Sociology of Education» es marcadamente diferente de la "vieja» en cuanto a sus contenidos, hay muy poco en ella que no se pueda encontrat ya en Durkheim y Mead o que no esté ya esbozado en el enfoque antetior. Consideremos, por ejemplo, el concepto de socialización (Gorbutt, 1974).

Para la sociología funcionalista, por ejemplo para T. Parsons, la socialización es un proceso a través del cual el niño es moldeado por la sociedad $o$, dicho de otro modo, adquiere las normas y valores que aseguren la perduración del sistema social. Recuérdese, en este mismo sentido, la célebre definición de educación de Durkheim. Se trata, evidentemente, de una visión «sobresocializada» del hombre y superintegrada de la sociedad (Wrong, 1961). El problema hobbesiano sobre el origen del orden y la cohesión sociales es explicado en un sentido mecanicista, es decir, a través de explícitas o implícitas referencias a la metáfota del alfarero: la interiorización de 
las normas y valores se realiza mediante un simple proceso de modelado de la conducta.

Pero frente a esta concepción mecanicista, que Berger ha titulado Ia analogía del títere, el nuevo concepto de socialización subraya que las relaciones entre el hombre y la sociedad son esencialmente dinámicas y dialécticas. Sus presupuestos antropológicos subyacentes destacan la dimensión recíproca del proceso de socialización: los socializados socializan a su vez a los agentes socializadores. E1 hombre es, desde esta nueva perspectiva, un agente activo que constraye la realidad social dando sentido a su mundo. El significado de hombres y cosas debe ser activamente interpretado y negociado. Además, la socialización es un proceso que se desarrolla a lo largo de todo el ciclo vital y que se produce en todo tipo de situaciones (no sólo en la familiar o la escuela, sino también en la empresa o el cuartel).

Del mismo modo, los antiguos temas de igualdad de oportunidades o «educabilidad» son tedefinidos a la luz de la sociología del conocimiento. De este modo, categorías que los educadores dan por supuestas («inteligencia», "buen alumno», «fracaso escolat»...) quedan abiertas a la investigación sociológica.

Aportes originales de la «New Sociology of Education». De acuetdo con Forquin (1983, p. 66 y ss.), entre los principales méritos de los «nue. vos» sociólogos cabe destacar su ampliación del campo o área de conocimiento en su triple sentido:

1. Teoría del curriculum. A su vez, subdivisible en el enfoque socio. estructural de Bernstein, para quien la educación es tanto reproducción cultural como control social, y en el enfoque socio-fenomenológico de Young, que enfatiza la arbitrariedad y el relativismo cultural de los saberes escolares.

2. Subjetivismo de los profesores. Crítica de las concepciones psicometristas, denuncia de la alienación pedagógica, etc.

3. Análisis de la interacción pedagógica. Por ejemplo, el estudio de los procesos de «etiquetado» en las relaciones profesor-alumno.

Más controvertidas son, en cambio, las innovaciones metodológicas que aporta la «New Sociology of Education». Ya be indicado que el nuevo pa" radigma es, fundamentalmente, un paradigma interpretativo muy estrechamente ligado a Ia sociología del conocimiento. Por consiguiente, si la dominación y el control son mucho más sutiles de lo que se suponía, y si el objetivo prioritario de la investigación sociológica es desmontar las teorías implícitas que se utilizan en las escuelas y cuestionar los modelos del «sentido común»... las herramientas tradicionales (sondeos de opinión, estadís- 
ticas, cuestionarios, etc.) no nos sirven. En este sentido, el nuevo paradigma es antipositivista, antibistórico y, predominantemente, microsociologico. Influenciados por la fenomenología (Berger y Lukmann, 1967), la etnometodología (Cicourel, 1967) y la interacción simbólica (Becker, 1952), les preocupa más la televancia de sus investigaciones que la precisión y exactitud de los datos que utilizan. Gran parte de su producción es estrictamente teórica, pero en su investigación empirica se muestran firmes partidarios de la metodología cualitativa (etnografía, estudio de casos, entrevistas en profundidad, registros en vídeo de la interacción didáctica...). Por otra parte, denuncian las supuestas «objetividad» $\mathrm{y}$ 《neutralidad» (a value free science) del «método científico». Según Karabel y Halsey $(1979$, p. 57), la «New Sociology of Education» posee el mérito de haber desmontado el mito de la cuantificación como criterio exclusivo de cientificidad; pero su hipercriticismo metodológico se convierte, de hecho, en una invitación a los discursos especulativos y subjetivistas.

Resulta prácticamente imposible recoger la catarata de críticas, usualmente adversas, que desde las diferentes ciencias de la educación y aun desde la propia sociología ha recibido la «New Sociology of Education» británica. Trataré de recoger alguna de las más significativas.

Desde el punto de vista metodológico cabe señalat su escasez de investigaciones empíricas (Shipman, 1973; Karabel y Halsey, 1977, etc.), su carácter eminentemente especulativo (Demaine, 1982) y su excesiva carga ideológica, que se ha traducido en una politización de la disciplina (Banks, 1982). A un nivel teórico y desde posiciones internas (o, cuanto menos, fronterizas) a la propia «New Sociology of Education», Williamson (1974) le ha reprochado su carácter exclusivamente microsociológico y anhistórico, hasta el punto de definirla como una «psicología social tadical». Por otra parte cabe señalar su tendencia a descuidar los condicionamientos estructurales y materiales que afectan tanto a los contenidos pedagógicos como a la interacción en el aula (Apple, 1978). Shipman (1973), aun admitiendo la validez de su descripción «desde dentro» del mundo escolar, le reprocha su dogmatismo y presunción. Además, pese a sus autoalabanzas, su enfoque es más descriptivo que propiamente interpretativo (Hurn, 1976).

No obstante, las críticas más severas, procedentes primordialmente de flósofos y pedagogos (Forquin, 1983), se centran en el llamado «igualitarismo epistemológico» de la "New Sociology of Education». En efecto, el enfoque ultrarrelativista y radical de los «nuevos» sociólogos conduce a una abolición de categorías tales como enseñantes-enseñados, éxito-fracaso, saber científico-saber vulgar... Si toda pedagogía es arbitraria, si cada individuo es libre de definit subjetivamente su propia realidad... cómo evitar caet en una actitud nihilista? Puestas así las cosas, la «New Sociology of Edu- 
cation» sólo puede conducir a la «desorientación pedagógica» (Forquin, 1983, p. 69) o al «igualitarismo sentimental» (Karabel y Halsey, 1979, p. 57).

En mi opinión, una adecuada evaluación de la «New Sociology of Education» británica debe considerar tanto el marco social y político en que surge el nuevo enfoque como las vicisitudes de su desartollo, especialmente las dimensiones institucionales del mismo. Como ha escrito Williamson (1974, p. 8), la aparición de estas nuevas tendencias no puede ser divorciada del supuesto fracaso de las reformas educativas de los años sesenta. En efecto, ni la educación compensatoria, ni la ampliación de la enseñanza universitaria ni la integración escolar... se tradujeron en una real disminución de las desigualdades educativas. Por ejemplo, sólo un tercio de las escuelas denominadas comprebensive eran de hecho escuelas integradas (Robinson, 1983; Shaw, 1981)... Bernstein (1970), por su parte, replicaba a quienes malinterpretando su obra ${ }^{7}$ confundian «código restringido» con déficit psicolingiústico. Denunciaba que se pretendiera que las reformas escolares fueran capaces de modificar las desigualdades sociales al tiempo que señalaba la dimensión ideológica de la «teoría del déficit» en la medida en que distrae la atención sobre las propias deficiencias de la institución escolar.

No sólo se siente el fracaso de la "vieja» sociología para presentar altetnativas viables, sino que se duda incluso de la capacidad del propio reformismo democrático para erosionar la estructura de poder del sistema. Recordemos, en efecto, que a finales de los sesenta las universidades europeas y americanas (París, Berkeley, Berlín...) se vieron sacudidas por un vendaval de movimientos estudiantiles radicales. Este clima de contestación estudiantil y de propuestas de contracultura puede explicar, junto a la atmósfera de fracaso del teformismo escolar, muchas de las actitudes subyacentes a la «New Sociology of Education»: igualitarismo, telativismo, nihilismo... En este sentido conviene no desestimar la dimensión generacional de la tuptura en el seno de la comunidad científica, ya que excepto los dos líderes M. F. D. Young y B. Bernstein, la gran masa de los «nuevos» sociólogos están cronológicamente muy próximos, cuando no forman parte integrante, de la generación de estudiantes contestatarios.

7. Obviamente, la obra de Basil Bernstein merecería por sí sola todo un extenso estudio monográfico. Tan sólo quisiera subrayar la visión caricaturesca, reduccionista y parcial que se acostumbra a tener de este importante sociólogo del que sólo se suele conocer, deformada y mal interpretada, su teoría de Ios códigos. Como excepción cabe citat: Alonso, I., Bernstein en la encrucijada de la Sociología de la Educacion, «Rev. Española de Investigaciones Sociológicasm, 11, julio/septiembre 1980, pp. 55-74.

8. Su autodefinición como RECKERS (Radical Egalitarian Critics of Knowledge and Educational Reality) es sumamente indicativa. 
A nivel institucional el nuevo paradigma debe refugiarse en los linderos del reducto académico: los colleges e institutos de educación. La «ciencia oficial» (léase los departamentos de sociología de las universidades) se muestra hostil $y$, durante bastante tiempo, la enseñanza de «New Sociology of Education» se verá confinada a las escuelas universitarias de magisterio. Según Halsey (Karabel y Halsey, 1979, pp. 50.51), la escasez de fondos de investigación en tales centros y su relativamente bajo estatus científico pudieran explicar, en parte, algunas de las particularidades metodológicas del nuevo enfoque; pot ejemplo, su predilección por la observación participante. En otras palabras, la utilización de las técnicas del survey research escapaba a las posibilidades económicas e institucionales de los centros universitatios donde mayoritariamente se difundió el nuevo enfoque; y ello tal vez explique su aversión a las metodologías cuantitativas. Estas dificultades institucionales explicarian, a mi modo de ver, la arrogancia y la agresividad del nuevo paradigma. Para decirlo con otras palabras: la recepción de un nuevo paradigma requiere muchas veces una redefinición de la ciencia correspondiente. Esta redefinición, todos lo sabemos, nunca es meramente teórica, sino que implica cambios en la distribución del poder, bienes económicos y prestigio y las consiguientes confrontaciones entre atacantes y defensores del statu quo académico.

Los dos centros difusores de la «New Sociology of Education» fueron el Instituto de Educación de Londres, donde estaba B. Bernstein desde 1963, y la Open University, donde se instalaton algunos de los más destacados discípulos de M. F. D. Young. El papel de los colleges de educación y de las escuelas de magisterio, aunque cuantitativamente impottante, posee menor relevancia teórica. En opinión de Williamson $(1974$, p. 10) la ubicación institucional del nuevo enfoque en centros vinculados a la formación de maestros explicaría muchas de sus limitaciones teóricas. Explicaría, por ejemplo, su descuido de las dimensiones histórica y macrosociológica del sis" tema educativo.

Llegados a este punto tal vez fueta conveniente replantearse el tema de si la «New Sociology of Education» constituye un paradigma alternativo y, si no es así, cuál es su significado. Obviamente la mayoría de sus cultivadores, e incluso gran parte de sus detractores, no dudaría en responder afirmativamente a la cuestión planteada. Además, se ha destacado en las páginas precedentes su ruptura epistemológica a nivel de los temas a considerar, de su estrategia metodológica e incluso de sus presupuestos filosóficos. Sin embatgo, ¿hasta qué punto se trata de un paradigma unitario? ¿En qué medida, por ejemplo, la obra de B. Bernstein se corresponde con lo que hasta ahora hemos considerado «New Sociology of Education»?

Parece evidente que Bernstein, pot seguir con el mismo ejemplo, está 
más próximo a una sociología estructuralista o neodurkheimniana (Atkinson, 1980) que a la fenomenología o al interaccionismo simbólico. Análogamente, dentro de la propia "New Sociology of Education» coexisten, desde sus inicios, enfoques interaccionistas y estructuralistas, especialmente marxistas. Dicha heterogeneidad teórica y conceptual se itá acrecentando, como veremos, en sus apottaciones más recientes. De hecho, y aun admitiendo la existencia de algunas características comunes entre sus seguidotes (Bernstein, 1977, p. 163), creo que deberíamos considerax la «New Sociology of Education» no como un paradigma unitario, sino como una plural y necesaria reacción contra la hegemonía del estructural-funcionalismo. Como ba indicado Forquin (1983, p. 73), la «New Sociology of Educations ha cumplicio durante una serie de años una función renovadora, polémica y programática. Su apologética imagen de marca ha abierto nuevos caminos a la sociología y ha demostrado que eran posibles otros nuevos enfoques antes de acabar diseminándose y fundiéndose en lo que podríamos llamar la ciencia sociológica «notmal». En las próximas páginas analizaremos la realidad presente de este enfoque, así como algunas de las testantes tendencias teóricas de la actualidad.

\subsection{Algunas tendencias recientes}

La reconversión de gran parte de la «New Sociology of Education» a un enfoque neomarxista obedecería, segín Banks (1982), a la propia endeblez teórica de dicho enfoque y, más concretamente, a su incapacidad para relacionar las microestructuras con las macroestructuras. Según esta misma autora (Banks, 1982, p. 24) en la actualidad no habria dos sino tres enfoques dominantes en la sociología de la educación británica. De hecho el asunto es tremendamente complejo, ya que, además de existir diferentes paradigmas, éstos suelen subdividirse en subáreas de conocimiento especializado (Davies, 1983). Además, el hecho de que la actual sociología de la educación tienda a tomar prestados conceptos y metodologías procedentes de otras disciplinas (historia de la educación, antropología cultural...) redunda en una mayor heterogeneidad de los diferentes enfoques (Demaine, 1982).

Destaquemos, en primer lugar, la resurrección de la «aritmética política» y el redescubrimiento de la noción de la «educabilidad» (Banks, 1982; Robinson, 1983). Obviamente, detrás de dicho resurgimiento hay una serie de condicionamientos sociopolíticos (crisis económica, «thatcherismo»...) que no podemos ignorar. Por supuesto, el pesimistno y el nithilismo de la "New Sociology of Education» la condenaron a una vigencia extraacadémica efímera al tiempo que abrieron el camino al redescubrimiento de la «vieja» 
«Nuevos» y «viejos» paradigmas en la sociología de la educación

sociología de la educación. Para decirlo en palabras de Williamson (1983), cuanto más crítica había llegado a ser la materia frente a sus propios métodos y supuestos, menos efectiva fue para producir cambios en la educación o en la sociedad. El divorcio creciente entre una sociología de la educación universitaria acumulativamente crítica y empíxicamente estéril y una investigación educativa crecientemente obsoleta y butocratizada, amén del cambio en el escenario político y económico, han vuelto a poner de actualidad al funcionalismo reformista.

El más conocido ejemplo de tal resurgit lo constituye el estudio de Halsey, Heath y Ridge (1980) sobre orígenes y destinos sociales. Sus resultados, fruto de un minucioso aparato estadístico, sugieren la persistencia de las desigualdades sociales y educativas a través de generaciones sucesivas. Pese a los cambios socio-económicos apenas se ha modificado, nos dicen, la estructura de posiciones telativas, como si de una escalera mecánica se tra. tara.

Por otra parte, se han detectado nuevas e importantes fuentes de desigualdades educativas: las diferencias geográficas (comarcas deprimidas, conflicto norte-sur...) y, especiaimente, el tema de la división sexual del trabajo. En efecto, el tema del género ${ }^{\circ}$ como condicionante de las desigualdades educativas goza actualmente de tal predicamento que está eclipsando al tradicional interés por las diferencias socio-económicas. Desde una perspectiva feminista, Acker (1981) ha denunciado el sesgo androcentrista de la sociología de la educación «nueva» y «vieja», tanto en lo que hace teferencia a la elección de temas como a la composición de las muestras, estatus aca. démico de las sociólogas, etc. Ia mayoría de estos estudios, predominante. mente realizados por sociólogas, versan sobre el papel de la escuela en el aprendizaje de los roles de sexo (Lobban, 1978; Delamont, 1980...), la persistencia de la discriminación sexual (Buswell, 1981), los estereotipos y las ideologías que los sustentan, la socialización sexual (Chetwynd y Hartnett, 1978), etc. Desde el punto de vista teórico (Arnot, 1981; Hartmann, 1981) cabe señalar dos grandes orientaciones: una perspectiva de economia política, marxista, y otra perspectiva culturalista. La primera, inspirada en la célebre obra de Engels sobre el origen de la familia, está centrada en los orígenes de la división sexual del trabajo y en el marco socio-económico que la sustenta. Al segundo enfoque no le interesa el «pot qué» de las diferencias del género sino el «cómo» son creadas y mantenidas... Análogamente, mien. tras la perspectiva marxista es predominantemente macrosociológica, la co-

9. Podemos definir al género como el modelo de conducta que una sociedad prescribe en función de la categorización sexual. Es clave la distinción entre sexo (criterio biológico) y géneto (criterio sociológico). 
rriente culturalista ha sido criticada por su incapacidad para explicar la dimensión socio-histórica del género, sus variaciones intelectuales y sus conflictos. De ahí el éxito creciente del concepto, impreciso pero movilizador, de "patriatcado".

Otra subespecialidad de la sociología de la educación británica que goza de continuada vigencia, especialmente a nivel de la formación de maestros, es el análisis de la interacción didáctica (Stubbs y Delamont, 1976; Delamont, 1981; Delamont, 1983...). Los ya clásicos estudios de Hargreaves en la década de los sesenta, como los más recientes análisis de etnografía del aula, recibieron, hasta hace aproximadamente una década, poca atención por parte de la sociología de la educación "oficial». Su carácter interdisciplinatio (en los Estados Unidos suelen ser practicados por antropólogos mientras que en ottos países, como Francia, se consideran ámbito de la psicología social...) y su difícil institucionalización académica no son óbice para su creciente importancia y difusión.

En relación con la actual crisis de la «New Sociology of Education» y su conexión con el enfoque denominado neomarxista, conviene precisar que la influencia del marxismo ya era perceptible en las primetas manifestaciones de este enfoque. No obstante, un análisis de contenido de los readings editados por la Open University nos evidencia el efecto acumulativo de tal acción..$^{10}$ ¿Qué ha ocurrido para que, como señala irónicamente Hickox (1982, p. 564), podamos ver al león marxista en aparente armonía con el cordero de la «New Sociology of Education»? Para este autor se tratatía de un proceso de reacciones en cadena: el reformismo funcionalista surgió como reacción frente al tradicionalismo pedagógico, las teorías tadicales suponen una primera reacción frente a las limitaciones de la sociología reformista y el neomarxísmo trata de corregir el «tomanticismo» de los «nuevos» sociólogos. Análogamente, Robinson (1983) atribuye la reconceptualización de la «New Sociology of Education» a la crítica que Gintis y otros sociólogos «comprometidos» hicieron del relativismo y subjetivismo de los presupuestos iniciales del nuevo enfoque. Bates (1980) arguye que la fenomenología puede proporcionar buenos atgumentos para atacar las diversas estratificaciones

10. En los dos primeros de estos readings (Cosin et al. eds., 1971; Hammersley y Woods, 1976) tanto los encabezamientos de los capítulos («Teaching and leaming as the construction of reality», «The culture of the school and the teacher's presentation of self», «Pupil cultures»...) como la mayoría de Ios autores seleccionados (Blumer, Schutz, Becker, Berger, Matza...) denotan el impacto de Ia sociología fenomeno16́gica y del interaccionismo simbólico. Por el contrario, los títulos del tercero de estos textos («Schooling and Capitalism», Dale et al. eds., 1976) y de la nueva antología («Education and the State»), así como la inclusión de autores como Gintis, Gramsci, Bowles, Marcuse, Willis... son expresión de la gradual sustitución del enfoque microsociológico inicial por nuevas posiciones teóricas próximas a la sociología marxista. 
del conocimiento y de la sociedad, pero falla en la defensa de cualquier otro sistema alternativo. Conviene, por tanto, cortegir las orientaciones iniciales hacia un nuevo enfoque que permita una teoría social crítica y una práctica efectiva. Por otra parte, la nueva epistemología no debe basarse en la racionalidad científica sino en nuevos criterios de validación: la efectividad de la acción con relación a su capacidad para mejorar la situación de la humanidad (Bates, 1980, p. 75).

La unovísima» sociología de la educación se distancia tanto del subjetivismo inicial de la «New Sociology of Education» como del estructuralismo de anteriores enfoques marxistas (Baudelot, Bowles y Gintis, Althusser...). Es decir, está más próxima a una sociología de la resistencia que a una sociología de la reproducción (Giroux, 1983). El acento se coloca en las tensiones y conflictos que median las relaciones entre el hogar, la escuela y el lugar de trabajo. Admite la relativa autonomía del sistema educativo frente a la infraestructura socio-económica y se preocupa por otras desigualdades educativas (géneto, etricidad, tesidencia...) además de las socio-económicas. Pot ejemplo, Paul Willis (1976) ha estudiado, combinando un enfoque tebrico marxista con una metodología etnográfica, la contracultura escolar de los adolescentes de clase trabajadora. Análogamente, Carol Buswell (1980) denuncia la descualificación profesional de un grupo de maestros por la introducción de una serie de cambios pedagógicos «innovadores». Aunque la recogida de datos se basa en la observación participante y en el uso de informantes, su marco de referencia teórico es también sociología marxista.

Para otros autores, el énfasis de la sociología de la educación debe colocarse en una metodología histótica y comparativa, por lo que nos temiten al «segundo» Durkheim (el de L'Évolution pedagogique...) y, especialmente, a Max Weber. Como representantes de este enfoque cabe citar al norteamericano Randall Collins (1971, 1979), así como a los británicos Margaret Archer y Bill Williamson. Para este último (Williamson, 1982) es preciso combinar sociología e historia para poder integtar tanto los aspectos estructurales como los interactivos de la vida social. Para los neoweberianos, las relaciones entre educación, cultura y estratificación deben ser enfocadas desde una perspectiva de sociología histórica.

Esta misma vocación de síntesis entre los niveles micro y macrosociológicos, aunque desde una posición neodurkheimniana, podemos observarla en la obra reciente de Basil Bernstein (1983).

En resumen, la expansión de la sociología de la educación británica ha sido tanto cuantitativa como cualitativa en la medida en que no sólo ha aumentado el número de cursos, estudiantes y profesotes, sino que también se ha ampliado el área de conociniento con la inclusión de diferentes perspectivas teóricas, innovaciones metodológicas y nuevos contenidos (Banks, 
1982, p. 27). Además, desde un punto de vista institucional (publicación de revistas especializadas, teconocimiento académico, inclusión en los planes de estudio...) ha alcanzado un notable grado de desarrollo... sólo amenazado por la virulencia de los conflictos entre paradigmas rivales. Aunque, como ha escrito Apple (1978, p. 495), si la medida de la vitalidad de una área de conocimiento es su intensidad de debate, entonces la sociología de la educación debe ser una disciplina muy saludable.

\section{A MODO DE CONCLUSION}

¿En qué medida es generalizable el proceso británico a la situación es. pañola? Obviamente resulta un tanto iluso equiparar la crítica situación de la sociología de la educación española con el rico aunque complejo panorama británico. En otras palabras, si su crisis es meramente de crecimiento, la nuestra es de nacimiento.

Paradólicamente, nuestra deficiente institucionalización de la disciplina se traduce en una serie de heterocronías de desarrollo. El problema, a mi modo de ver, no es tanto nuestro retraso (tanto a nivel de elaboración teó. rica como, muy especialmente, en lo referente a la investigación) como las incoherencias de nuestro proceso de institucionalización. Por ejemplo, mientras los británicos, pese a su riqueza de enfoques teóricos y al artaigo académico de esta área de conocimiento, se conforman con celebrar una reunión anual (en el Westhill College de la Universidad de Birmingham), nosotros, debido tal vez a nuestra tiqueza turística, solemos celebrar tres y hasta cuatro veces al año, y en los más variados escenarios geográficos, «jornadas», «semanas» y congresos relacionados con eí tema.

Por otra parte, nuestro sectarismo (no tanto teórico como sencillamente corporativista) nada tiene que envidiar a los enfrentamientos entre «viejos» y «nuevos» sociólogos británicos. Tampoco nuestro nihilismo y afán desmitificador. Parece, en efecto, como si pretendiéramos «estar de vuelta» aun antes de haber alcanzado el umbral. ¿Se trata de «paradojas del oficio» (Moncada, 1983) o, por el contrario, de un frívolo mimetismo?

Necesitamos no sólo superar el ensayismo retórico y los conflictos gremialistas, sino aprender a reconocer como positiva la pluralidad teórica. En este sentido creo que debemos aceptar la conveniencia de incluir planteamientos «hetetodoxos» y de favorecer las relaciones interdisciplinarias, salvo el caso de que nuestra pretensión sea reducir la sociología a una amalgama de religión y política...; una mezcla tan anacrónica como peligrosa. 
«Nuevos» y «viejos» paradigmas en la sociología de la educación

\section{BIBLIOGRAFIA}

Ackers, S. (1981): No-Woman's-land: British Sociology of Education 1960-1979, «Sociological Review», 29 (1), pp. 77-104.

Alonso, I. (1983): Centro y periferia en Sociología de la Educación: su desigual $e$ incierto proceso de institucionalización, «Rev. Española de Investigaciones Sociológicas», 24, pp. 163-181.

Apple, M. W. (1978): The New Sociology of Education: Analyzing Cultural and Economic Reproduction, «Harvard Educational Review», 48 (4), páginas 495-503.

Arnot, M. (1981): Culture and Political Economy: Dual Perspectives in the Sociology of Women's Education, "Educational Analysis», 3 (1), pp. 19.36.

Atkinson, P. (1980): «Bernstein's Structuralism», mimeografiado, Dep. of So ciology, University College of Cardiff.

Banks, O. (1962): The Sociology of Education, 1952-1982, «British Journal of Educational Studies», 30 (1), pp. 18-31.

Bates, R. J. (1980): New Developments in the New Sociology of Education, «British Journal of Sociology of Education», 1 (1), pp. 67.79.

Becker, H. S. (1952): The career of the Cbicago public scbool teacher, en Hammersley, M. y P. Woods, eds. (1976), ob. cit., pp. 75-80.

Berger, P. L. y T. Luckmann (1967): La construcción social de la realidad, Amotrortu, Buenos Aires, 1973.

Bernstein, B. (1970): «Education cannot compensate for society», en Cosin, B. R. et allii eds. (1971), ob. cit., pp. $64-69$.

- (1971): «On the classification and framing of educational Knowledge», en M. F. D. Young (1971) ed., reimpreso en Bernstein, B., Class, Codes and Control. Towards a Theory of Educational Transmissions, Routledge and Kegan Paul, Londres, 1975, pp. 85-115.

- (1975): «The Sociology of Education: a bref account», en Bernstein, B. (1975), pp. 157-165.

- (1983): «Educational Transmissions», Seminario realizado en la Esc. Universitaria de Magisterio de Barcelona (Universidad de Barcelona), febrero 1983.

Buswell, C. (1980): Pedagogic Change and Social Change, «British Journal of Sociology of Education», 1 (3), pp. 293-306. 
- (1981): Sexism in Scbool Routines and Clasroom Practices, «Durham and Newcastle Research Review», X (46), pp. 197-200.

Byrne, D. y B. Williamson (1975): The poverty of Education, Martin Robertson, Londres.

Cicourel, A. (1964): El método y la medida en sociologia, Editora Nacional, Madrid, 1982.

Collins, R. (1971): «Funcional and Conflict Theories of Educational Stratification", en Cosin, B. R., ed., Education: Structure and Society, Penguin, Harmondsworth, 1972, pp. 175-199.

- (1979): The Credential Society, Academic Press, Londres.

Cosin, B., R. Irdale, G. M. Esland, D. Mackinnon y D. F. Swift, eds. (1971): Scbool and Society, Open University Press, Londres.

Chetwynd, D. y O. Hartnett, eds. (1978): The Sex Role System, Routledge and Kegan, Londres.

Dale, R., G. Esland y M. Mac Donald (1976): Scbooling and Capitalism, Open University Press, Londres.

Davies, A. (1976): Social control and Education, Methuen, Londres.

- (1982): «Sociology and the sociology of education», en Hartnett, A., ed, The Social Sciences in educational Studies, Heinemann, Londres, pp. 33-52.

- (1983): «Tendencias en el desartollo de la Sociología de la Educación en el mundo anglosajons, en VV.AA. (1983): Perspectivas actuales en Sociología de la Educación, I.C.E. de la Univ. Autónoma de Madrid, Cantoblanco.

Delamont, S. (1980): Sex roles and the School, Methuen, Londres.

- (1981): All too familiar? A decade of classroom research, «Educational Analysis», 3 (1), pp. 67-100.

- (1983): Interaction in the Classroom, Methuen, Londres.

Demaine, $J$. (1982): Contemporary Tbeories in the Sociology of Education, Macmillan, Londres.

Forquin, J. C. (1983): La «nouvelle sociologie de l'éducation» en Grande-Bretagne: oriéntations, apports théoriques, évolution (1970-1980), «Revue Française de Pedagogies, 63, pp. 61-79.

Giroux, H. A. (1983): Theories of Reproduction and Resistance in the New Sociology of Education: A Critical Analysis, «Harvard Educational Review», 53 (3), pp. 257-293.

Gorbutt, D. (1974): «The New Sociology of Education», en Reid, I. y E. Wormald, eds. (1979), ob. cit., pp. 98-106.

Halsey, A. H. (1965): «Sociology for Teachers», en Reid, I. y E. Wormald, eds. (1979), ob. cit., pp. 29-32.

Hammersley, M. y P. Woods (1976): The Process of Schooling, Open University Press, Londres.

Hartmann, H. (1981): «The Unhappy Marriage of Marxism and Feminism: Towards a more progresive union", en Dale, R. et alii, eds., Education and the State (II). Politics, Patriarchy and Practice, Falmer Press, Londres, 1981, pp. $191-210$. 
Hickox, M. S. H. (1982): The Marxist sociology of education: a critique, «The British Journal of Sociology», 33 (4), pp. 536-578.

Hurn, C. J. (1976): Recent Trends in the Sociology of Education in Britain, «Harvard Educational Review», 46 (1), pp. 105.114.

Karabel, J, y A. H. Halsey (1977): «Educational Research: A Review and an Interpretation», en Karabel, J. y A. H. Halsey, eds., Power and Ideology in Education, Oxford University Press, Nueva York, 1979, pp. 1-86.

Keddie, N. (1971): «Classroom Knowledge», en M. F. D. Young, ed. (1971), ob. cit., pp. 133-160.

Lerena, C. (1983): «Sociología y Ciencias de la Educación». Ponencia leída en las II Jornadas de Sociología de la Educación, Escuelas Universitarias de Magisterio, Granada, mayo 1983.

Lobban, G. (1978): «The Influence of the School on Sex-Role Stereotyping», en Chetwynd, J. y O. Hartnett (1978), ob. cit., pp. 50-61.

Mannheim, K. (1936): Ideologia y utopia, Aguilar, Madrid, 1966.

- (1943): «La educación, la sociología y el problema de la ciencia social», en Mannheim, K., Diagnóstico de nuestro tiempo, Fondo de Cultura Económica, México, 1966, pp. 78-86.

March, M. X. (1984): «La Sociología de la Educación: entre la crisis, el fanatismo, la repetición y el desencanton, comunicación presentada en el Segun. do Congreso de Saciologia (FASSEE), Santander, septiembre 1984.

Mascaró, J. (1983): «Sociología de la Educación. Nuevas Direcciones», en VV.AA., Diccionario de las Ciencias de la Educación, Santillana, Madrid, vol. II, pp. 1.316-1.317.

Moncada, A. (1983): Los usos de la sociologia de la educación, «Sociología», 2, pp. 3-7.

Musgrave, P. W. (1965): «Sociology in the Training of Teachers», en Reid, I. y E. Wormald, eds. (1974), pp. 33.40.

Ortega, F. (1983): «Pasado y presente de la sociología de la educación en España», en VV.AA. (1983), ob. cit., pp. 347-375.

Pegram, M. y B. Abell (1974): "An Analysis of Sociology main course sylabuses in Colleges of Educations, en Reid, I. y E. Wormald (1974), eds., pp. 15-26.

Quintana, J. M. (1983): Por una bistoria de la Sociología de la Educación en España, «Revista Española de Pedagogía», 159, pp. 25-42.

Reid, Y. (1974): «Sociology in Colleges of Education: Some Considetations», en Reid, I. y E. Wormald, eds. (1974).

Reid, I. y E. Wormald, eds. (1974): Sociology and Teacher Education, Association of Teachers in Colleges and Departments of Education, Londres.

Robinson, P. (1983): "Whatever happened to educability?», mimeografiado, University of Keele.

Sanvicens, A. (1969): «Constantes y coincidencias pedagógico-sociológicas en la evolución cultural europea», VI Semana Internacional de Estudios Sociales, «Las constantes de Europa», Instituto de C. Sociales, Barcelona, 1969, vol. II, pp. $479-529$.

Shaw, B. (1981): Educational Practice and Sociology, Martin Robertson, Oxford. 
Shipman, M. D. (1973): Bias in the Sociology of Education, «Educational Review», 25 (3), pp. 190-200.

Stubbs, M. y S. Delamont, eds. (1976): Explorations in Classroom Observation, Wiley, Chichester.

Subirats, M. (1983): Sociología de la educacion, "Cuadernos de Pedagogía», 100, pp. $79-84$.

Szereter, R. (1980): Institutionalising a New Specialism: early years of the Journal of Educational Sociology, «British Journal of Sociology of Education», 1 (2), pp. 173-182.

- (1983): Writing and Writer in Britisb Sociology Periodicals, 1953-1979, «British Journal of Sociology of Education», 4 (2), pp. 155-168.

Williamson, B. (1974): «Continuities and Discontinuities in the Sociology of Education», en Flude, M. y J. A. Hier, Educability, Scbools and Ideology, Halsted Press, Nueva York, pp. 2-14.

- (1982): Class, Culture and Community, Routledge and Kegan Paul, Londres.

- (1983): «Conocer ¿para qué?: el contexto de la investigación en la Sociología de la Educación», en VV.AA. (1983), ob. cit., pp. 49-66.

Willis, P. (1976): «The class significance of school counter-culture», en Hamersley, M. y P. Woods, eds. (1976), ob. cit., pp. 188.199.

Wrong, D. H. (1961): The Oversocialized Conception of Man in Modern Sociology, «Ametican Sociological Review», 26 (2), pp. 183-193.

Young, M. F. D., ed. (1971): Knowledge and Control New Directions for the Sociology of Education, Collier-Macmillan, Londres. 